\title{
UTILISATION D'UN CAROTÉNOIDE DE SYNTHĖSE COMME MARQUEUR NUTRITIONNEL POUR L'OMBLE DE FONTAINE (S. FONTINALIS) ELLEVÉ EN PISCICULTURE
}

\author{
A. GUIllou*, **, J. DE LA NOUE* **
}

* Centre de recherche en nutrition, Pavillon Comtois, Cité universitaire, Université Laval, Ste-Foy, Québec, Canada, G1K 7P4.

* Département de biologie, Pavillon Vachon, Cité universitaire, Université Laval, Ste-Foy, Québec, Canada, G1K 7P4.

\section{RESUME}

Un marquage nutritionnel des ombles de fontaine $(20-23 \mathrm{~cm})$ de pisciculture peut être réalisé grâce à l'ester éthylique de l'acide $\beta$-apo-8'-caroténoïque (EEAC), caroténoïde de synthèse, qui est absent de la chair d'ombles sauvages contrairement à la canthaxanthine. Après 4 et 6 semaines d'alimentation avec un aliment contenant $100 \mu \mathrm{g} \mathrm{EEAC/g}$, la majorité des individus commercialisables $(150 \mathrm{~g}$ et plus) montrent de faibles teneurs en EEAC dans le muscle. De plus, la coloration obtenue avec la canthaxanthine de synthèse chez les ombles marqués n'est pas différente de celle des ombles témoins. Dans le contexte de la commercialisation de l'omble de fontaine d'élevage au Québec (Canada), ce type de marquage, peu coûteux, peut constituer un moyen de prévention de l'augmentation possible du braconnage d'individus sauvages.

\section{THE USE OF A SYNTHETIC CAROTENOID AS A NUTRITIONAL MARKER FOR FARMED BROOK TROUT (S. FONTINALIS)}

\section{ABSTRACT}

It is possible to differenciate farmed brook trout $(20-23 \mathrm{~cm})$ from wild ones by the incorporation of $\beta$-apo-8'-carotenoic acid ethyl ester (CAEE) in the diet. In contrast with canthaxanthin this synthetic carotenoid is absent from wild trout flesh. After 4 and 6 -week feeding periods with a meal containing $100 \mu \mathrm{g}$ of CAEE $/ \mathrm{g}$, most trout of marketable size ( $150 \mathrm{~g}$ and more) show small, but significant muscular CAEE concentration. Moreover there is no difference in flesh coloration between experimentally marked trout and controls.

This inexpensive marker could be used to prevent a possible increase of poaching on wild brook trout induced by the marketing of this native Québec (Canada) species.

\section{INTRODUCTION}

La mise sur le marché québécois de l'omble de fontaine d'élevage risque d'entraîner une recrudescence du braconnage de l'omble sauvage. Un marquage des individus de pisciculture s'impose dès lors pour éviter toute contestation.

Le marquage nutritionnel des ombles de fontaine présente des avantages indéniables sur les autres formes de marquage ; car les poissons ne sont pas stressés ou blessés par des manipulations; une fois l'aliment préparé, il n'y a pas de travail ou de coûts supplémentaires; le marquage des poissons se fait en même temps que la pigmentation des chairs.

La non toxicité, des analyses rapides et peu coûteuses de même qu'un coût acceptable de l'aliment marqué sont les conditions que doit respecter le marqueur retenu. L'ester éthylique de l'acide $\beta$-apo-8'-caroténoïque (EEAC) est un caroténoïde de synthèse ; on ne le retrouve pas dans la chair d'ombles sauvages, contrairement à la canthaxanthine (BILODEAU, communication personnelle). II possède un fort pouvoir colorant (FERRANDO et MAINGUY, 1970) et est déjà ajouté à l'alimentation animale pour accentuer la coloration de la chair de poulet et du jaune d'œuf (BAUERFEIND, 1972). L'EEAC est aussi utilisé dans l'industrie alimentaire pour colorer différents types d'aliments (pâtes alimentaires, pâtisseries, jus de fruits, etc.). II est accepté comme additif alimentaire par la loi canadienne sur les aliments et les drogues ainsi que dans plusieurs pays. La concentration limite permise est de $35 \mu \mathrm{g} / \mathrm{g}$ de poids sec dans la nourriture destinée à la consommation humaine. 
L'objet du présent travail est de mesurer les limites de l'utilisation de ce pigment comme marqueur nutritionnel des ombles de fontaine élevés en pisciculture.

\section{MATERIEL ET METHODES}

L'expérience a été menée pendant huit semaines à l'Aquarium de Québec. Quatre-vingt-dix ombles de fontaine de 1 an + , d'un poids moyen de $136 \mathrm{~g}$. ont été introduits dans trois bassins cylindriques de $70 \mathrm{~cm}$ de diamètre (200 litres) ayant chacun un débit d'eau (circuit ouvert) de 10 litres/min.

De I'EEAC à deux concentrations (100 et $200 \mu \mathrm{g} / \mathrm{g}$ d'aliment sec) est ajouté à un aliment de type commercial contenant $200 \mu \mathrm{g} / \mathrm{g}$ de canthaxanthine/g. L'incorporation du pigment se fait par mélange homogène avec l'aliment broyé, réhydratation partielle, passage à la filière et déshydratation à $35^{\circ} \mathrm{C}$ pendant 12 heures. Les aliments (expérimental et témoin) sont conservés au congélateur a $-10^{\circ} \mathrm{C}$. Les deux concentrations utilisées ont été retenues d'après les résultats d'une étude préliminaire.

Les poissons sont acclimatés pendant deux semaines aux conditions expérimentales. Durant l'expérience, la température de l'eau est de $11^{\circ} \mathrm{C}$. et la photopériode naturelle. La nourriture est distribuée manuellement, une fois par jour, à un taux de $1 \%$ du poids vif. Après quatre semaines complètes d'alimentation, treize poissons par bassin sont prélevés au hasard pour fins d'analyses. Après deux semaines supplémentaires d'alimentation, treize autres individus par bassin sont, à leur tour, prélevés au hasard. Les ombles sont congelés pendant deux mois à $-20^{\circ} \mathrm{C}$. Une bande de $5 \mathrm{~g}$ de chair dépourvue de peau est prélevée au-dessus de la ligne latérale pour l'extraction des caroténoïdes totaux selon la méthode AOAC (1980).

Les extraits de caroténoïdes sont purifiés par élution sous vide dans des colonnes à chromatographie de $12 \mathrm{~mm} \times 50 \mathrm{~cm}$ dont l'adsorbant est un mélange 1:1 de silicagel G et de Hyflo Supercell (Fisher).

La récupération des caroténoïdes est faite par rinçaye des colonnes avec $30 \mathrm{ml}$ d'une solution d'hexane-acétone (80:20). Après évaporation complète du solvant sous vide, les caroténoïdes sont gardés à l'obscurité dans des ballons scellés contenant de l'azote, à une température de $-20^{\circ} \mathrm{C}$. pendant deux jours. La totalité des caroténoïdes extraits de la chair est reprise dans $5 \mathrm{ml}$ de phase mobile (acétonitrile :7; dichlorométhane:2; méthanol:1) et dosés sur HPLC d'après la méthode décrite par NELLS et DE LEENHEER (1983).

L'analyse de la coloration des chairs est faite à partir de $\mathbf{5}$ g de chair débarrassée de la peau et prélevée au-dessus de la ligne latérale de chacun des individus. Les bandes de chair sont finement hachées et homogénéisées dans $5 \mathrm{ml}$ d'eau distillée. Trois lectures de réflectance (colorimètre Pacific Scientific modèle Colorigard system/05) sont prises sur l'homogénat; la moyenne donne la valeur numérique de la coloration des échantillons de chair.

\section{RESULTATS}

La figure 1 montre l'évolution de la concentration des caroténoïdes musculaires. On note une tendance générale à l'augmentation de la concentration des pigments (canthaxanthine, EEAC et son dérivé non identifié) avec l'augmentation du poids des individus pour le groupe nourri pendant six semaines avec $100 \mu \mathrm{g}$ d'EEAC/g d'aliment.

Le tableau 1 présente la concentration moyenne de l'EEAC et de son dérivé ainsi que celle de la canthaxanthine retrouvées chez les groupes expérimentaux et témoins. Les teneurs d'EEAC musculaires sont faibles comparativement à celles obtenues pour la canthaxanthine. Le meilleur résultat de marquage est obtenu avec $100 \mu \mathrm{g}$ d'EEAC/g d'aliment après une période d'alimentation de six semaines, puisque les quantités de marqueur incorporé ainsi que le nombre des individus marqués sont plus élevés qu'après quatre semaines d'alimentation.

La figure 2 représente les valeurs colorimétriques des échantillons de chair des individus. Nous constatons une plus grande dispersion des résultats selon l'axe de la quantité relative de rouge que selon l'axe de la quantité relative de vert (jaune).

\section{DISCUSSION}

\section{Concentration des caroténoïdes dans le muscle :}

Il faut souligner que la méthode utilisée pour l'extraction des caroténoïdes totaux produit un dérivé non identifié de I'EEAC (Fig. 1). La méthode utilisée pour obtenir les standards donne le pigment pur, mais lorsque la méthode d'extraction est utilisée avec l'EEAC sous forme commerciale, on détecte la présence de ce dérivé en HPLC. II est très semblable à l'EEAC, puisque son temps de lecture précède celui-ci de quelques secondes. II peut être aussi considéré comme un marqueur, car on ne le retrouve pas dans la chair des ombles témoins. 
Les résultats rapportés à la figure 1 sont appuyés par des expériences menées avec des truites arc-en-ciel. Il est généralement accepté que la fixation musculaire de la canthaxanthine est meilleure chez les sujets les plus lourds TORRISSEN et NAEVDAL, 1983) et efficace seulement à partir de $150 \mathrm{~g}$ (KOENIG, 1975 ; ABDUL MALAK, 1975).

Seuls les ombles de $150 \mathrm{~g}$ et plus sont commercialisables, puisque sous ce poids ils ne sont pas considérés comme "portion" par les marchands qui sont alors moins enclins à les acheter. Pour cette raison, seuls les individus d'un poids supérieur à $150 \mathrm{~g}$ au terme de la période de marquage pris au hasard ont été retenus pour analyse. A cause des forts écarts types, on n'observe pas de différence significative entre les concentrations d'EEAC et de canthaxanthine dosées dans la chair des ombles des groupes expérimentaux (Tab. 1).

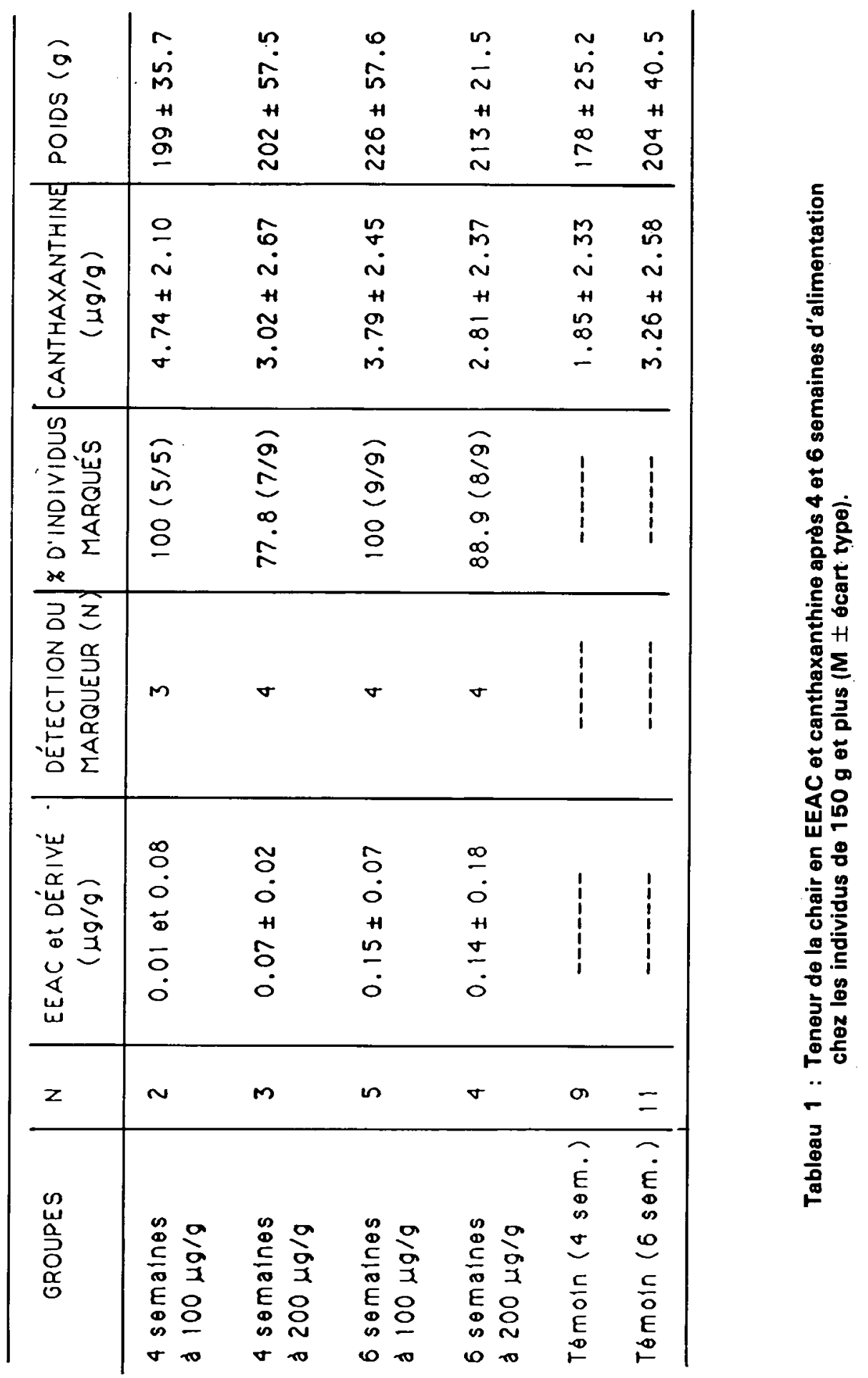




\section{Concentration d'EEAC et son dérivé}

\section{$(\mu g / g)$}

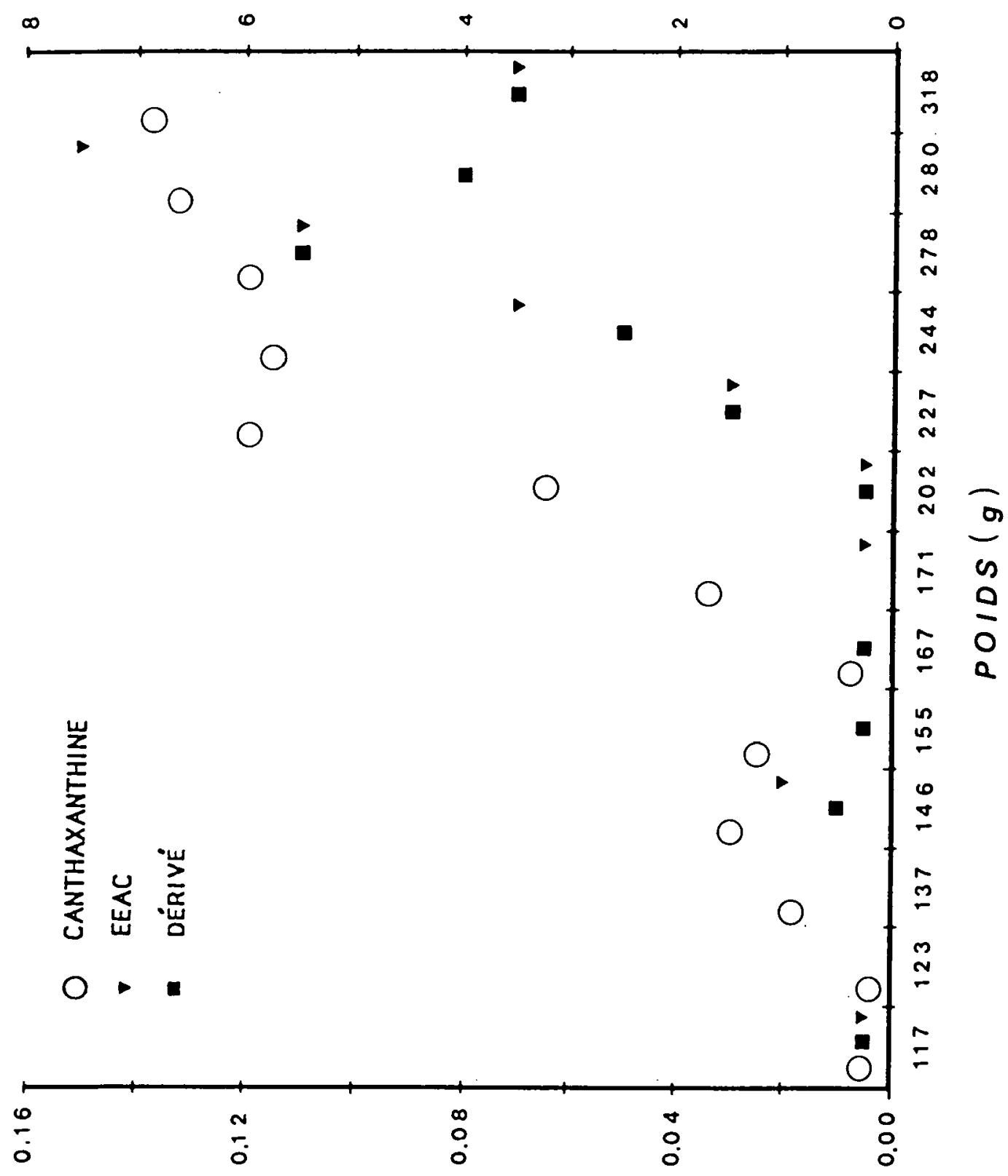

$(6 / 6 \pi)$

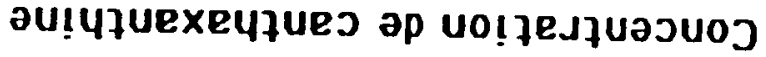

Figure 1 : Concentration des caroténoïdes musculaires en fonction du poids des individus nourris pendant 6 semaines avec un aliment contenant $100 \mu \mathrm{g} \mathrm{d}^{\prime} \mathrm{EEAC} / \mathrm{g}$.

Figure 1 : Concentration of muscular carotenoids for fish fed diets containing $100 \mu \mathrm{g}$ of $\mathrm{CAEE} / \mathrm{g}$ for a 6-week period. 


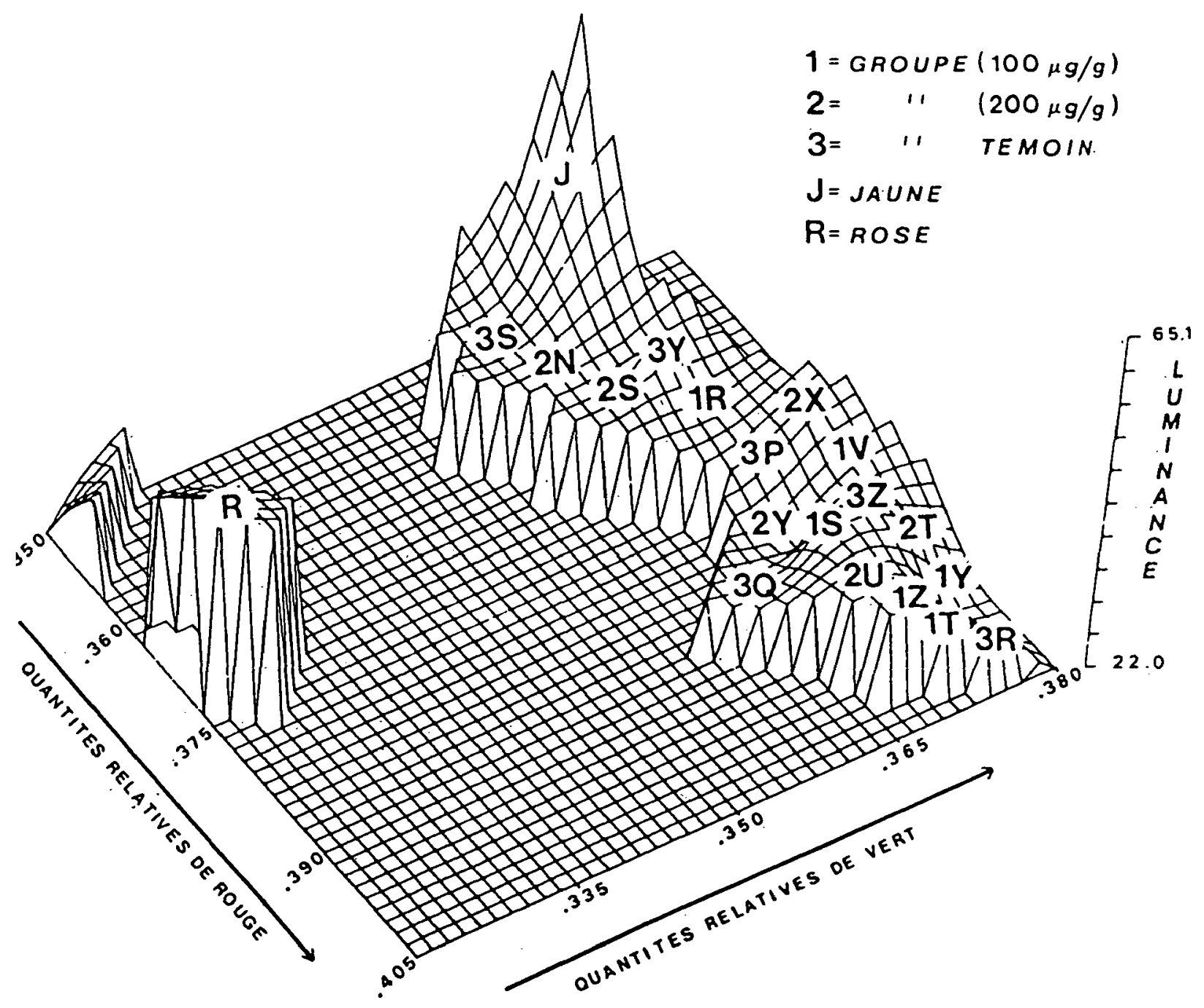

Figure 2: Couleur des chairs après 6 semaines d'alimentation. Les lettres associées aux groupes 1 (100 $\mu \mathrm{g}$ d'EEAC). 2 (200 $\mu \mathrm{g}$ d'EEAC) et 3 (canthaxanthine seulement) représentent les individus possédant une coloration de la chair recherchée par les commerçants.

Figure 2 : The colour of fish flesh after a 6-week feeding period. The letters associated with groups 1 (100 $\mu \mathrm{g}$ of CAEE). 2 (200 $\mu \mathrm{g}$ of CAEE) and 3 (canthaxanthin only) represent fish showing a marketable flesh colouration.

A notre connaissance il n'y a pas de travail publié sur l'utilisation de l'EEAC chez le poisson. II est donc difficile de discuter des facteurs influençant la fixation de ce pigment chez l'omble de fontaine. D'autre part, plusieurs facteurs identifiés aujourd'hui peuvent influencer la fixation de la canthaxanthine (CHOUBERT, 1981) et, par analogie, probablemént aussi celle de l'EEAC dans le muscle des salmonidés. Les principaux sont une déficience dans l'alimentation des individus dominés causée par la forte hiérarchie des ombles dans une colonne d'eau (bassins cylindriques) et la maturité sexuelle des poissons. Lors de la maturation des gonades des femelles, les pigments caroténoïdes y sont emmagasinés au détriment du muscle (STEVEN, 1949 ; SHNAREVICH et SAKHNENKO, 1971. : TACON, 1981). Chez les mâles ie transfert de pigments se ferait vers la peau (CROZIER, 1969). De plus, la variation dans les aptitúdes génétiques à fixer le pigment canthaxanthine chez la truite arc-en-ciel est connue (KOENIG, 1975); TORRISSEN et NAEVDAL, 1983; BLANC et CHOUBERT, 1985). L'état de santé des poissons joue probablement aussi dans cette fixation (KOENIG, 1975) et ceci est d'autant plus vrai pour l'EEAC, puisqu'il est un précurseur de la 
vitamine A (BRUBACHER, 1972). Le sexe des individus joue également, les mâles possédant une moindre aptitude à la pigmentation que les femelles (KOENIG, 1975). Par contre, TORRISSEN et NAEVDAL (1983) ne trouvent aucune différence significative de fixation de la canthaxanthine selon le sexe chez la truite arc-en-ciel.

La période de reproduction de l'omble de fontaine se déroule normalement du début de décembre à la fin de janvier. En effet, quelques ombles de chaque groupe montraient un développement complet des gonades. Cette variation dans le développement des gonades des ombles expérimentaux explique probablement une bonne partie des variations observées dans la quantité de pigments fixés dans le muscle ainsi que l'absence de marqueur chez trois individus de plus de $150 \mathrm{~g}$ (groupes alimentés avec $200 \mu \mathrm{g}$ d'EEAC/g d'aliment pendant 4 et 6 semaines; voir tab. 1). On peut penser qu'un marquage estival (ombles de 1 an+) produirait de moins grandes variations dans la teneur des pigments fixés, puisqu'à cette période de l'année, les gonades sont très peu développées chez les ombles de cette classe d'âge.

\section{Effets du marqueur sur la coloration du muscle :}

La concentration des deux formes du marqueur (EEAC et dérivé) est faible par rapport à celles de la canthaxanthine (Tab. 1), ce qui laisse présager du faible effet qu'auront ces marqueurs sur la coloration de la chair des ombles expérimentaux.

Pour l'appréciation de la couleur des chairs, nous avons, dans un premier temps, classé à l'œil nu la couleur des chairs obtenues après les périodes d'alimentation en cinq catégories: blanche, jaunâtre, orangé pâle, orangé moyen et orangé foncé. L'appréciation était subjective, mais permettait néanmoins de constater que la saturation de la coloration variait généralement en fonction du poids des individus, tandis que la teinte de la chair des sujets bien alimentés n'était pas affectée par la présence ou l'absence du marqueur. Nous avons utilisé le système des coordonnées $X, Y, Z$ de la Commission Internationale de l'Eclairage (CIE) comme méthode objective de mesure de la couleur de la chair d'omble. La luminance est maximale à 86.2 (blanc parfait) et minimale à 0.0 (noir); donc, plus la luminance d'un échantillon est faible plus la saturation de la couleur est grande. La figure 2 montre bien que la plus grande source de variation dans la teinte des différents échantillons de chair survient selon l'axe de la quantité relative de rouge ce qui n'est pas le cas pour l'axe vert (jaune). Etant donné que la canthaxanthine est un pigment rcuge et que I'EEAC est jaune, il est ainsi confirmé que la principale cause de variation de la coloration des individus marqués est due aux variations interindividuelles dans la fixation musculaire de la canthaxanthine. Nous observons aussi un chevauchement de plusieurs plages représentant la couleur des échantillons des trois groupes (expérimentaux et témoin); nous pouvons alors conclure qu'il n'y a pas de différence appréciable de la couleur entre les individus des groupes expérimentaux et ceux du groupe témoin possédant des concentrations semblables de canthaxanthine (Fig. 2). II est à noter que des résultats semblables ont été obtenus avec les ombles marqués pendant 4 semaines.

Le coût de revient de l'aliment marqué avec I'EEAC est un aspect important qui conditionne la rentabilité de son utilisation. L'EEAC sous sa forme commerciale coûte actuellement $90 \$$ can. $/ \mathrm{kg}$; pour obtenir une concentration de $100 \mu \mathrm{g}$ de pigment $/ \mathrm{g}$ d'aliment, il faut ajouter $1 \mathrm{~g} / \mathrm{kg}$ d'EEAC commercial à l'aliment (pigment pur $=10 \%$ d'EEAC sous forme commerciale). II en coûterait donc 1,80 s can. de plus par sac de $20 \mathrm{~kg}$, soit une augmentation de $7,20 \%$ durant la période de marquage. Ce coût supplémentaire semble élevé à première vue, mais reste néanmoins raisonnable lorsqu'il est réparti sur toute la période d'élevage des poissons, soit environ un an et demi. En effet, la période de marquage des ombles de fontaine n'excède pas huit semaines, ce qui représente seulement $13 \%$ de la période de croissance des individus destinés au marché de l'alimentation. II en coûtera donc environ $1 \%$ de plus au producteur pour obtenir des ombles de fontaine marqués.

\section{REMERCIEMENTS}

Nous remercions le personnel de l'Aquarium de Québec, particulièrement le directeur M. PAULHUS ainsi que M. LESSARD. Nous désirons remercier aussi M.BILODEAU (MAPAQ), pour ses conseils et son expertise technique, M. CHOUBERT (I.N.R.A., France) pour ses avis éclairés ainsi que la compagnie HOFFMANN-LA ROCHE pour les échantillons de caroténoïdes. Les travaux ont été financés par le Conseil des Recherches et Services Agricoles du Québec (CRSAQ).

\section{BIBLIOGRAPHIE}

ABDUL MALAK N., 1975, Influence de certains facteurs nutritionnels et écologiques sur le métabolisme d'un pigment caroténoïde : la canthaxanthine, chez la truite (Salmo gairdneri R.). Thèse de Doctorat $3^{\circ}$ cycle, Lyon, $106 \mathrm{p}$.

A.O.A.C. Methods of analysis, 13th ed. 1980. Carotens and xanthophyls in dried plant materials and mixed feeds. Method 43018, p. 739. 
BAUERFEIND J.C.. 1972. Carotenoid vitamin A precursors and analogs in foods and feeds. J. Agr. Food Chem., $20: 456-472$.

BLANC J.M., CHOUBERT G., 1985. Genetic variation of flesh colour in canthaxanthine fed rainbow trout. Génét. Sél. Envol., 17 (2), 243-250.

BILODEAU M., 1983. Communications personnelles.

BRUBACHER G.B., 1972. L'importance des caroténoïdes en alimentation animale. Documentation Roche, édité par F. Hoffman - La Roche Cie - 10, rue Crillon. Paris IVe.

CHOUBERT G., 1981. Caroténoïdes et pigmentation. In Nutrition des poissons. FONTAINE (ed.), Actes Coll. CNERNA, PARIS Mai, 1979, CNRS, PARIS, 283-295.

CROZIER G.F., 1970. Tissue carotenoids in prespawning and spawning sockeye salmon (Oncorhynchus nerka). J. Fish. Res. Bd. Can., 27, 973-975.

FERRANDO R., MAINGUY P., 1970. La couleur du poulet de chair, compte rendu d'expérimentation. Documentation Roche, édité par F. Hoffmann-La Roche Cie - 10, rue Crillon, Paris.

KOENIG J., 1975. Pigmentation de la truite. Documentation Roche, édité par F. La Roche Cie - 10, rue Crillon, Paris.

NELLS H.J.C.F., DE LEENHEER A.P., 1983. Isocratic nonaqueous reversed-phase liquid chromatography of carotenoids. Analytica Chem. $55: 270-275$.

SHNAREVICH I.D., SAKHNENKO E.G., 1971. Dynamics of carotenoids in tissue and organ of fish relative to the sexual cycle (russe). Gidrobiol. Zh., 7. 90-93.

STEVEN D.M., 1948. Studies on animal carotenoids. I. Carotenoids of the brown trout (Salmo trutta Linn.) J. Exp. Biol., 25, 369-387.

TACON A.G.V., 1981. Speculative review of possible carotenoid function in fish. Prog. Fish Cult., 43: 205-208.

TORRISSEN O.J., NAEVDAL G., 1983. Pigmentation of salmonids - Genetical variation in carotenoid deposition in Rainbow trout. Aquaculture, 38 : 59-66. 